\title{
Comparison of Jupiter interior models derived from first-principles simulations
}

\author{
B. Militzer • W.B. Hubbard
}

Received: 23 June 2008 / Accepted: 25 November 2008 / Published online: 5 December 2008

(C) The Author(s) 2008. This article is published with open access at Springerlink.com

\begin{abstract}
Recently two groups used first-principles computer simulations to model Jupiter's interior. While both studies relied on the same simulation technique, density functional molecular dynamics, the groups derived very different conclusions. In particular estimates for the size of Jupiter's core and the metallicity of its hydrogen-helium mantle differed substantially. In this paper, we discuss the differences of the approaches and give an explanation for the differing conclusions.
\end{abstract}

Keywords Equation of state - First-principles simulations · Density functional theory $\cdot$ Hydrogen-helium mixtures . Giant planet interiors

The characterization of the interior structure of giant planets in our solar system is crucial for identifying their formation mechanism and for understanding the evolution of the solar system. Establishing the history of our solar system will help interpreting the observed similarities and differences between our and other solar systems. The unexpected diversity among the over three hundred recently discovered extra solar planets has challenged existing theories of planetary formation and migration.

The planets in our solar system have been studied in great detail with observations and space missions but many

\footnotetext{
B. Militzer $(\bowtie)$

Departments of Earth and Planetary Science and of Astronomy, University of California, Berkeley, CA 94720, USA

e-mail: militzer@berkeley.edu
}

W.B. Hubbard

Lunar and Planetary Laboratory, The University of Arizona,

Tucson, AZ 85721, USA questions about their interior structures have remained unanswered. Jupiter is predicted to have a relatively small rocky core of between zero and seven Earth masses (Saumon et al. 1995; Saumon and Guillot 2004), which is surprising because similar theories predicted between 10 and 25 Earth masses for the core in Saturn (Saumon and Guillot 2004). This prediction for Jupiter has lent support to coreaccretion theories with comparatively small cores (Pollack et al. 1996), late-stage core erosion scenarios (Guillot 2004), or suggesting that Jovian planets are able to form directly from gases without a triggering core (Boss 2007).

The small core hypothesis for Jupiter has now been challenged in a paper by Militzer, Hubbard, Vorberger, Tamblyn, and Bonev (MHVTB) (Militzer et al. 2008) who used firstprinciples computer simulations of hydrogen-helium mixtures to compute the equation of state (EOS) in the interior of Jupiter. This work predicts a large core of 14-18 Earth masses for Jupiter, which is in line with estimates for Saturn and suggests that both planets may have formed by coreaccretion. The paper further predicts small fraction of planetary ices in Jupiter's envelope suggesting that the ices were incorporated into the core during formation rather than accreted along with the gas envelope. Jupiter is predicted to have an isentropic and fully convective envelope that is of constant chemical composition. In order to match the observed gravitational moment $J_{4}$, the authors suggest that Jupiter may not rotate as a solid body and predicted the existence of deep winds in the interior leading to differential rotation on cylinders.

The first-principles simulations used in the MHVTB paper are a major difference compared to chemical EOS models developed by Saumon, Chabrier, and van Horn (Saumon and Chabrier 1992; Saumon et al. 1995). With first-principles simulations one simulates a fully interacting quantum system of over a hundred electrons and nuclei and therefore 
avoids a number of approximations used in chemical models. In chemical models one for example describes hydrogen as an ensemble of stable molecules, atoms, free electrons, and protons and is then required to make additional approximations to treat their interactions. These approximations are dependent on the material under consideration and may also depend on the temperature and pressure. Firstprinciples methods describe the interactions on a fundamental level. One is also required to make some approximations to solve the many-body Schrödinger equation but those are very different in nature, are not material specific and do not depend on the $T$ and $P$. Therefore one expects EOSs derived from first-principles to be more accurate than chemical models unless they have been fit to experiments. However, no experimental EOS data are available for the deep interiors of giant gas planets.

The MHVTB paper described significant differences between the first-principles EOS and chemical models. However these were not solely responsible for the different predictions for Jupiter's interior. In a different paper, Nettelmann, Holst, Kietzmann, French, Redmer and Blaschke (NHKFRB) Nettelmann et al. (2008) also used first-principles method to study Jupiter's interior but came to very different conclusions. Very much in line with earlier models, they predict a small core for Jupiter and a large amount of heavy elements in the envelope.

In this paper, we will objectively discuss the differences between the MHVTB and NHKFRB approaches in order to explain how such different conclusions were derived, although we encourage the reader to compare the two original papers also. The differences between the two papers can be sorted into three categories: (1) differences in DFT-MD simulations, (2) differences in the subsequent construction of adiabats, and (3) different assumptions in the models for Jupiter's interior. We will go through these differences in the following three sections and demonstrate that the main difference arises from point (3).

\section{Comparison of simulation parameters}

In this section we discuss the differences in the firstprinciples simulations performed by the two groups. Although there are differences in the level of accuracy, they are unlikely to be the main reason for the differences in the Jupiter models. Both groups used density functional molecular dynamics (DFT-MD) simulations and should derive identical equations of state for hydrogen, helium, and their mixtures. However, computational details are important for accuracy of the derived EOS.

The NHKFRB group used exclusively VASP code while MHVTB used CPMD as well as the VASP code. MHVTB verified that both codes yield identical results when run accurately.
The MHVTB group performed simulations of hydrogenhelium mixtures using a mixing ratio that closely resembles Jupiter's composition within the limitations of a finite simulation cell. The NHKFRB instead relied on the ideal mixing approximation using EOSs of pure hydrogen and helium. This approximation neglects all interactions between hydrogen and helium. In Vorberger et al. (2007a, 2007b), it was shown that this interaction leads to significant corrections in the derived pressures and energy but, more importantly, the presence of helium increases that stability of the hydrogen molecules for given $T$ and $P$. These interaction effects also have an impact on the derived adiabat that will be discussed in the next section. However, linear mixing approximation does not affect the predicted core mass very much. In fact, the MHVTB group used their own set of simulations for pure hydrogen and helium (Militzer 2008) in a separate Jupiter model and derived a core mass that differed by only 2 Earth masses. MHVTB use this independent calculation to estimate the uncertainty of the predictions.

The NHKFRB group performed DFT-MD simulations with 500-2000 time steps. MHVTB performed simulations for 2 picoseconds with 5000 time steps. Longer simulations lead to more accurate averages for thermodynamic variables such as pressure and internal energy.

Concerning the size of the simulation, the NHKFRB paper states that simulations with between 32 and 162 atoms for hydrogen, helium, and water were performed. A conservative estimate of the precision of the EOS $5 \%$ is given. Conference presentations by this group showed results with 64 hydrogen atoms and 32 helium atoms (64 electrons). The MHVTB group used simulations with 128 electrons throughout, which are more accurate.

The NHKFRB used $\left(\frac{1}{4}, \frac{1}{4}, \frac{1}{4}\right)$ Baldereschi point to sample the Brillouin zone for all simulations. The MHVTB group analyzed the $k$-point dependence using simulation with up to $4 \times 4 \times 4 k$-points and then performed simulation with $2 \times 2 \times 2$ in the metallic regime and using the $\Gamma$ point at lower densities. It was shown that $\Gamma$ point only simulation with 128 electrons overestimate the pressure near Jupiter's core-mantle boundary by $1.6 \%$.

\section{Derivation of the adiabats}

Convection dominates the heat transport in giant gas planets. This leads to an adiabatic temperature-pressure profile for the planet's interior with the exception of a small low pressure region near the surface where radiative heat transport takes over. It is not clear if the cores of giant planets themselves are convective but the following planetary models are insensitive to the temperature profile in the core. On the other hand, the temperature profile in the hydrogenhelium rich envelope and the required derivation of adiabats 
are very important to characterize the interiors of giant planets. However, neither Monte Carlo nor molecular dynamics methods can compute the entropy directly because both techniques save orders of magnitude of computer time by generating only a representative sample of configurations instead of integrating over the whole configuration space that would be needed to derive the entropy.

One typically derives the entropy by thermodynamic integration from a known reference state. This can be very computationally demanding and is also not needed to map out planetary interiors. The absolute value of the entropy is not important as long as one is able to construct $(T, P)$ curves of constant entropy. This can be achieved by using the pressure and the internal energy from first-principles simulations at different $(T, V)$ conditions. Using Maxwell's relations, one finds,

$\left.\frac{\partial T}{\partial V}\right|_{S}=-\frac{\left.\frac{\partial S}{\partial V}\right|_{T}}{\left.\frac{\partial S}{\partial T}\right|_{V}}=-T \frac{\left.\frac{\partial P}{\partial T}\right|_{V}}{\left.\frac{\partial E}{\partial T}\right|_{V}}$

By solving this ordinary differential equation, $(V, T)$ adiabats can be constructed as long as a sufficiently dense mesh of high-quality EOS points are available to make the required interpolation and differentiation of $E$ and $P$ with respect to temperature satisfactorily accurate.

One drawback of formula (1) is that it is not necessarily thermodynamically consistent if pressures and internal energies are interpolated separately. This is the primary reason why we developed a fit for the free energy (Militzer 2008) that is thermodynamically consistent by construction. The free energy, $F(V, T)$, is fit in such a way that first-principles pressures and internal energies are reproduced by,

$P=-\left.\frac{\partial F}{\partial V}\right|_{T} \quad$ and $\quad E=F-\left.T \frac{\partial F}{\partial T}\right|_{V}$.

The details of this method are described in Militzer (2008) where a helium EOS derived from first-principles simulations is presented for a large temperature and pressure interval.

We apply this fit across the insulator-to-metal transition in fluid hydrogen-helium mixtures (Chabrier et al. 2006). According to predictions from the best simulation methods currently available, quantum Monte Carlo (Delaney et al. 2006) and DFT-MD (Vorberger et al. 2007a) this transition is expected to occur gradually. However, for pure hydrogen, the dissociation transitions gives raise to a region of negative $\partial P /\left.\partial T\right|_{V}$ (Bagnier et al. 2000; Vorberger et al. 2007a), which leads to a negative Grüneisen parameter and might introduce a barrier into Jupiter's convection. However, the MHVTB work demonstrated that in a hydrogen-helium mixture the region of $\partial P /\left.\partial T\right|_{V}<0$ is shifted to lower temperatures than occur in Jupiter. Significant effort went into analyzing this transition within the DFT-MD method. The con- clusion is that Jupiter's interior is fully convective and this is main reason for constructing a two-layer model.

The two papers under consideration both use the BornOppenheimer MD (BOMD) technique where the electronic wave function is converged at every ionic step to obtain instantaneous ground state. Earlier DFT-MD simulations (Scandolo 2003; Bonev et al. 2004) instead used the Car-Parrinello (CP) method (Car and Parrinello 1985) to propagate the electronic wave functions. The key idea of the CP method is the construction of a fictitious dynamical system that couples ionic and electronic degrees of freedom to allow for very efficient updates of the wave function. It relies on a clear separation of electronic and ionic frequencies. For many materials, the method has been shown to give results that are in very good agreement with the more accurate but also more expensive BOMD method. However, dense hydrogen near the molecular-to-metallic transition is a special case where CPMD and BOMD differ. The CPMD method (Scandolo 2003; Bonev et al. 2004) predicts an abrupt dissociation transition while BOMD predicts it to be a continuous process.

At high pressure, Pauli exclusion leads the delocalization of electronic charge (Militzer 2005) and the electrons can no longer provide a sufficient binding force for the protons. In such an event the shape of the electronic orbitals is tightly coupled to the motion of the nuclei, and a clear separation of electronic and ionic frequencies no longer exists. That is reason why CPMD has difficulties predicting the dynamical properties of dense hydrogen near the dissociation transition. The fictitious dynamics is prone to deviate from the Born-Oppenheimer surface and to predict dissociation to occur too soon. One should rely on the BOMD technique instead.

The dissociation transition leads to a region where $\partial P /\left.\partial T\right|_{V}$ is small but positive. Following (1), the temperature along the adiabat raise very little in this region. As a result, the predicted temperatures for the metallic regime reaching all the way down to core-mantle boundary are significantly lower than predicted by the NHKFRB model. There is no information in the NHKFRB paper on how the entropy was derived. At this point, one cannot verify whether (1) is fulfilled. The NHKFRB adiabats agree well with adiabat from the $\mathrm{SCvH}$ EOS model for lowest and highest pressures in Jupiter's envelope. A temperature of $18600 \mathrm{~K}$ is predicted for the core-mantle boundary.

The MHVTB adiabats, on the other hand agree with $\mathrm{SCvH}$ only in the low density limit. In the region of molecular dissociation, the slope of the adiabats, $\partial T /\left.\partial \rho\right|_{S}$, is reduced, which leads to a lower temperature profile for the regime of metallic hydrogen. The MHVTB model predicts a temperature of $13300 \mathrm{~K}$ for the core-mantle boundary. This temperature is substantially lower than the NHKFRB prediction and more work will be needed to resolve the discrepancy. At Jupiter's core-mantle boundary the pressures 
are not yet high enough for the hydrogen-helium mixtures would be weakly interacting. To obtain an independent estimate for the entropy and therefore temperature, one would need to construct another path for thermodynamic integration.

\section{Comparison of Jupiter interior models}

The main difference between the two Jupiter's model under consideration arise from the modeling assumptions. The MHVTB group derived a new type of Jupiter model that has only two layers: a dense core and a completely isentropic (consistent with full convection and no phase transitions) mantle composed of mostly hydrogen and helium. Because there is no freedom in this type of model to redistribute mass by invoking chemical discontinuities in the mantle, the group could only match the gravity moment $J_{4}$ and all other constraints by invoking differential rotation with deep winds in Jupiter's envelope instead of the conventional solid-body rotation with minor surface winds. Substantial differential rotation in Jupiter, potentially detectable by the forthcoming Juno orbiter, was one of main predictions of the MHVTB paper.

The NHKFRB group used a three layer model that is much closer to previous models by Saumon and Guillot (2004). Besides a solid rock core, NHKFRB model assumes the mantle to be composed of two layers with differing composition. The flexibility to distribute helium and heavier elements unevenly in the two mantle layers and the associated redistribution of mass allows NHKFRB group to match $J_{4}$ without additional assumptions such as differential rotation. This is main difference between the two models. The redistribution of mass in the NHKFRB model to match $J_{4}$ also reduces the predicted core mass and leads to a larger amount of heavier elements in the envelope.

In the NHKFRB model, the inner layer of Jupiter is helium rich $(24.5 \%)$ and contains metallic hydrogen while the outer layer is comparatively helium poor (23.3\%) and contains molecular hydrogen. However, the NHKFRB model also predicts a very large difference in the concentration of heavier elements: $2.1 \%$ in the outer and $16.6 \%$ in inner layer. An explanation how such large concentration differences can arise during Jupiter's evolution remains to be given. In this regard, the MHVTB is much simpler. It assumes a fully convective mantle of constant chemical composition.

NHKFRB used the standard theory of figures to derive Jupiter's gravity field. In contrast, MHVTB used two independent approaches to derive the gravity field. The first approach used the theory of figures, while the second approach used the self-consistent-field method incorporating arbitrary differential rotation (Hubbard et al. 1975; Hubbard
1982) to monitor numerical errors in the gravity field calculations and to confirm that the $J_{4}$ mismatch for solidbody rotation is not a numerical artifact. Because NHKFRB used a standard solution for the uniformly-rotating polytrope of index one to test their code, it seems unlikely that their gravity calculations are affected by errors either. However, as a minor point, NHKFRB fitted their models to an older value for $J_{4}$ with larger error bars instead of using (Jacobson 2003).

Summarizing one can say that MHVTB predicted a large core in Jupiter of 14-18 Earth masses using a new twolayer model with a fully convective envelope. The NHKFRB work predicted a much smaller core based on a threelayer model that is very similar to earlier Jupiter models. The crucial difference lies in the treatment of the molecular-tometallic transition in dense fluid hydrogen and more work is needed to characterize this transition with different experimental and theoretical techniques. MHVTB analyzed this transition within density functional theory and concluded that this transition is continuous leading to an Jupiter envelope of constant chemical composition. Instead of analyzing this transition, NHKFRB follow previous models and made the additional assumption of two chemically different mantle layers. Besides the discussed accuracy differences in the computed EOS, we attribute this extra assumption to be the primary reason with the difference in the predicted core masses for Jupiter.

Acknowledgements We thank J. Vorberger and D. Stevenson for comments.

Open Access This article is distributed under the terms of the Creative Commons Attribution Noncommercial License which permits any noncommercial use, distribution, and reproduction in any medium, provided the original author(s) and source are credited.

\section{References}

Bagnier, S., Blottiau, P., Clarouin, J.: Phys. Rev. E 63, 015301 (2000) Bonev, S.A., Militzer, B., Galli, G.: Phys. Rev. B 69, 014101 (2004)

Boss, A.P.: Astrophys. J. 661, L73 (2007)

Car, R., Parrinello, M.: Phys. Rev. Lett. 55, 2471 (1985)

Chabrier, G., Saumon, D., Potekhin, A.Y.: J. Phys. A 39, 4411 (2006)

Delaney, K.T., Pierleoni, C., Ceperley, D.M.: Phys. Rev. Lett. 97, 235702 (2006)

Guillot, T., et al.: In: Bagenal, F., Dowling, W.M.T.E. (eds.) Jupiter Cambridge Univ. Press, Cambridge (2004) Chap. 3

Hubbard, W.B.: Icarus 52, 509 (1982)

Hubbard, W.B., Slattery, W.L., DeVito, C.L.: Astrophys. J. 199, 504 (1975)

Jacobson, R.A.: JUP230 orbit solution. http://ssd.jpl.nasa.gov/? gravity_fields_op (NASA-JPL document) (2003)

Militzer, B.: J. Low Temp. Phys. 139, 739 (2005)

Militzer, B.: Submitted to Phys. Rev. B. arXiv:0805.0317 (2008)

Militzer, B., Hubbard, W.H., Vorberger, J., Tamblyn, I., Bonev, S.A.: Astrophys. J. Lett. 688, L45 (2008)

Nettelmann, N., Holst, B., Kietzmann, A., French, M., Redmer, R., Blaschke, D.: Astrophys. J. 683, 1217 (2008) 
Pollack, J., Hubickyj, O., Bodenheimer, P., Lissauer, J.J., Podolak, M., Greenzweig, Y.: Icarus 124, 62 (1996)

Saumon, D., Chabrier, G.: Phys. Rev. A 46, 2084 (1992)

Saumon, D., Guillot, T.: Astrophys. J. 609, 1170 (2004)

Saumon, D., Chabrier, G., Horn, H.M.V.: Astrophys. J. Suppl. 99, 713 (1995)
Scandolo, S.: Proc. Natl. Acad. Sci. 100, 3051 (2003)

Vorberger, J., Tamblyn, I., Militzer, B., Bonev, S.: Phys. Rev. B 75, 024206 (2007a)

Vorberger, J., Tamblyn, I., Bonev, S., Militzer, B.: Contrib. Plasma Phys. 47, 375 (2007b) 